\title{
Individual vocal signatures in barn owl nestlings: does individual recognition have an adaptive role in sibling vocal competition?
}

\author{
A. N. DREISS, C. A. RUPPLI \& A. ROULIN \\ Department of Ecology and Evolution, University of Lausanne, Lausanne, Switzerland
}

Keywords:

acoustic communication;

begging:

bioacoustics;

condition;

genetic;

heritability;

identity:

recognition;

sex;

sibling negotiation;

vocal signature.

\begin{abstract}
To compete over limited parental resources, young animals communicate with their parents and siblings by producing honest vocal signals of need. Components of begging calls that are sensitive to food deprivation may honestly signal need, whereas other components may be associated with individualspecific attributes that do not change with time such as identity, sex, absolute age and hierarchy. In a sib-sib communication system where barn owl (Tyto alba) nestlings vocally negotiate priority access to food resources, we show that calls have individual signatures that are used by nestlings to recognize which siblings are motivated to compete, even if most vocalization features vary with hunger level. Nestlings were more identifiable when food-deprived than food-satiated, suggesting that vocal identity is emphasized when the benefit of winning a vocal contest is higher. In broods where siblings interact iteratively, we speculate that individual-specific signature permits siblings to verify that the most vocal individual in the absence of parents is the one that indeed perceived the food brought by parents. Individual recognition may also allow nestlings to associate identity with individual-specific characteristics such as position in the within-brood dominance hierarchy. Calls indeed revealed age hierarchy and to a lower extent sex and absolute age. Using a cross-fostering experimental design, we show that most acoustic features were related to the nest of origin (but not the nest of rearing), suggesting a genetic or an early developmental effect on the ontogeny of vocal signatures. To conclude, our study suggests that sibling competition has promoted the evolution of vocal behaviours that signal not only hunger level but also intrinsic individual characteristics such as identity, family, sex and age.
\end{abstract}

\section{Introduction}

Many social interactions observed in nature involve some forms of individual recognition (Tibbetts \& Dale, 2007). Recognizing counterparts can be particularly useful during long-lasting and repeated interactions with multiple individuals. For instance, in birds, singing males can vocally discriminate neighbours from strangers (Stoddard et al., 1991) and high-competitive from low-competitive opponents (Naguib \& Todt, 1997), and partners of a monogamous couple can recognize each

Correspondence: Amelie N. Dreiss, Department of Ecology and Evolution, University of Lausanne, Biophore, Lausanne CH-1015, Switzerland. Tel.: 00412169241 74; fax: 00412169241 65;

e-mail: amelie.dreiss@unil.ch

Correction added on 22 November 2013, after first online publication: grammatical error in title corrected. other (Lind et al., 1996). In family interactions, offspring vocal signatures allow nestmates to modify competitive behaviour according to kinship (Boncoraglio et al., 2009) and parents to recognize their progeny and avoid feeding alien offspring (Holley, 1984; Aubin \& Jouventin, 1998; Levrero et al., 2009). Because animal vocalization can not only reveal their identity but also signal motivation to compete and resource-holding potential, different vocal cues may be used to perform these multiple tasks (Reers \& Jacot, 2011). For instance, offspring begging calls can simultaneously advertise need for parental care (Christe et al., 1996; Marques et al., 2009) and identity (Levrero et al., 2009). Multiple vocal cues may thus permit to jointly indicate stable individual characteristics such as identity or sex and dynamic elements such as need for parental care, body or social condition (Tibbetts \& Dale, 2007). 
Few studies showed that young animals could be individually recognized by their parents or siblings. One of the few examples showed that juvenile Greylag Geese (Anser anser) can recognize one another (Scheiber et al., 2011), which may facilitate long-term social interactions that are sophisticated in this species. In some passerines, each parent takes care of only part of the brood which may relate to parental ability to discriminate individual offspring (Draganoiu et al., 2006). Although recognition may be based on individualspecific attributes, such a detailed system where each single individual can be efficiently distinguished is not always required. A less accurate recognition system may be sufficient if selection favours vocal signatures to allow animals to discriminate conspecifics only by kinship, familiarity, age or gender (Tibbetts \& Dale, 2007). Vocalizations of various animal species are indeed known to be influenced by genetic factors (Medvin et al., 1992; Forstmeier et al., 2009) or by learning (Jenkins, 1978; Janik \& Slater, 2000; Kedar et al., 2000) and could hence present a family signature. For instance, when young are provisioned after birth, brood recognition may be enough for parents to discriminate and feed their own young (but see Leonard et al., 2003). Although there are situations where young animals may be selected to be recognizable, especially in colonial species in which young are mobile (Holley, 1984; Aubin \& Jouventin, 1998), in other cases, hiding identity or origin may be beneficial, for instance for brood parasites or extra-pair young (Kempenaers \& Sheldon, 1996). Moreover, the benefit from signalling or hiding identity may fluctuate according to the context. For instance, bird begging calls were found more identifiable in hungry than in satiated chicks (Reers \& Jacot, 2011), suggesting that individuals produced more recognizable calls when highly motivated to win the vocal contest. We argue here that individual recognition may have evolved because it strengthens the honesty of signals, as it allows the signalling performance to be assigned to the correct individual. Recognition should be important if receivers face several signallers and assess their endurance over a long period of time (Payne \& Pagel, 1997). It should be also particularly important in communication systems where the response to signalling (such as mate choice by females or food allocation by parents) is delayed in time compared with the emission of the signal. In such systems, information gathered at signal emission about signallers' quality or motivation would be used later on when signal is no longer transmitted. Receivers would have to remember signallers' identity and signal level when performing a behavioural choice. We studied here individual signature in a 'sibling negotiation' system, in which sibling barn owls (Tyto alba) advertise their hunger level to nestmates during the long hours of parental absence, in order to be given the priority access to the food delivered afterwards, once parents are back (Roulin, 2002; Johnstone \& Roulin, 2003).

Barn owl nestlings are appropriate to test whether vocalization can simultaneously advertise identity, a component that remains stable across time, and need for parental care, a component that strongly varies through time and hence is flexible. In this system, the two to nine nestlings of a brood communicate among each other while parents are hunting far from the nest. Hungry nestlings inform their siblings in parent absence about their motivation to compete for the next food item to be delivered by their parents by emitting many and long calls (Roulin, 2002; Roulin et al., 2009). These vocal signals deter siblings from vocalizing and from competing for the prey at parental return, and hence, the most vocal nestling has priority access to the impending indivisible prey item without having to compete too intensely (Roulin, 2002; Dreiss et al., $2010 b)$. Communication before parent arrival would permit each nestling to optimally invest in competition for food at parent's return, according to the probability of monopolizing the next delivered prey item (Johnstone \& Roulin, 2003). This sib-sib communication system referred to as 'sibling negotiation' therefore reduces the cost of sibling competition and is expected to evolve when food resources are indivisible, that is, a single nestling is paid back for the effort invested in sibling competition (Johnstone \& Roulin, 2003). In dyadic sib-sib interactions, age hierarchy due to asynchronous hatching affects vocal production as less competitive junior nestlings have to produce more calls to defy their senior nestmates (Dreiss et al., 2010b). Interestingly, siblings also adjust their vocalization to the past vocalizations produced by nestmates (Dreiss et al., 2013a), indicating that they recognize individual siblings and remember their performance.

Current knowledge on this sib-sib communication process suggests that barn owl nestlings can concomitantly identify individual siblings and assess their level of need and competitive ability based on vocal cues (Dreiss et al., 2013a). In a first move into the study of such a complex system, we here examine three issues. First, we investigated the extent to which seven vocal parameters are related to hunger level, within-brood age hierarchy, gender and absolute age. Second, we statistically measured the degree with which we can discriminate individual siblings based on their negotiation calls and whether this discrimination varies with hunger level. Finally, using a cross-fostering experimental design, we tested whether these vocal parameters are related to the nest of origin (most likely due to genetic factors) and/or to the nest of rearing. To this end, we measured call rate and six acoustic parameters (related to frequency, loudness and within-call variation) in pairs of nestmates that could freely interact vocally. Nestlings from the same pair differed in age and were recorded during two nights: one night in a food-satiated 
state and one night in a food-deprived state. Because our experimental design did not allow us to determine whether nestlings discriminate individual siblings (although a previous paper showed that this is the case, Dreiss et al., 2013a), we asked students to recognize calls produced by two nestlings. The frequency discrimination and range of detected frequencies being comparable in barn owls and humans (Quine \& Konishi, 1974), this experiment permits to confirm the discriminability of siblings' call.

\section{Materials and methods}

\section{Study population and cross-fostering experiment}

We performed the study in 2008 in western Switzerland $\left(46^{\circ} 4^{\prime} \mathrm{N}, 6^{\circ} 5^{\prime} \mathrm{E}\right)$ on a population of wild barn owls breeding in nest boxes. Parents hunt small mammals at night to feed their one to nine offspring. Eggs are laid on average every 2.5 days, and incubation starts after the first egg has been laid generating a pronounced age hierarchy among siblings. Nestlings stay 2 months in the nest and hence have ample time to interact vocally with their nestmates. We estimated that in the absence of parents, a single nestling produces between 1000 and 5000 calls per night that are directed to siblings (Roulin, 2002).

We performed a cross-fostering experiment by exchanging an equal number of nestlings (1-3) between 19 pairs of nests on average $4.3 \pm 0.3$ days after hatching. We exchanged only nestlings of the same age, by comparing the length of their left flattened wing from the wrist to the tip of the longest primary (Roulin, 2004). Seven nests were not cross-fostered because no nestlings of the same age could be found. Consequently, 68 nestlings used to study vocal signatures were raised in a different nest ('nest of rearing') than the one where they were born ('nest of origin'), whereas 78 nestlings were raised in the nest where they were born. Nestmates can be considered as full siblings in the barn owl, because extra-pair paternity is rare (1 of 211 offspring was not sired by the social male, Roulin et al., 2004).

\section{Recording setup}

About 10 days before fledging takes place at approximately 55 days, we brought 22 - to 46-day-old nestlings to the laboratory where they were housed in a soundproof wooden nest-box $\left(62 \times 56 \times 37 \mathrm{~cm}^{3}\right)$, similar to the ones where they were reared under natural conditions. In the laboratory, we randomly matched in dyads nestlings coming from the same nest of rearing. We placed them in the same box but separated by a thin wooden wall pierced with five holes at the top, so that they could hear each other without visually or physically interacting. Each dyad of nestlings comprised a senior individual and a $5 \pm 0.5$ (SE) days younger junior nestmate (range in age difference: 1-15 days). Nestling vocalization could not be recorded spontaneously when isolated, as they hardly call in isolation, even when food-deprived (A. N. Dreiss, personal observation). For this reason, we only obtained vocalization data of pairs of nestlings. After a first night of acclimation, each dyad of siblings was recorded twice from the beginning of the night until midnight: one night in a food-deprived state (no food given to the two individuals during the preceding $28 \mathrm{~h}$ ) and another night in a food-satiated state (from 00:00 to 16:00 on the recording day, we offered $130 \mathrm{~g}$ of laboratory mice to each of the two individuals, which exceeds their daily food requirement of about $67 \mathrm{~g}$ ), with the order of the two treatments being randomly assigned across dyads. Food-deprived individuals lost on average $42 \pm 1 \mathrm{~g}$ over $24 \mathrm{~h}$, whereas they gained $16 \pm 2 \mathrm{~g}$ over $24 \mathrm{~h}$ when fed ad libitum. Individuals that were starved on the first night were randomly chosen, because their mean body mass at the start of the experiment was similar as the mean body mass of individuals receiving the ad libitum treatment the first night (Student's $t$-test: $\left.t_{202}=0.63, P=0.53\right)$. Ten of the 156 nestlings did not vocalize during the two 4.5 -h recording sessions, a situation that also occurs in natural conditions (personal observation). We hence analysed the vocalization of 146 nestlings including 76 males and 68 females and two individuals of unknown sex, from 43 broods. Thirty-eight nestlings vocalized only during the fooddeprived treatment and 10 only during the food-satiated treatment. Among the 73 dyads of nestlings from the same nest of rearing, 38 nestlings were from a different nest of origin and 35 were full siblings from the same nest of origin. Preliminary analysis showed that relatedness between nestlings (i.e. full-sibs or unrelated nestlings) of a pair did not affect any parameters of vocal production (not shown).

\section{Discrimination of barn owl calls by human ears}

In 2010, 20 students (11 women and nine men) aged 20-25 years were asked to determine whether a call was produced by the same or a different nestling than the previous call of a broadcast sequence. Each student listened to eight successive sequences of 40 calls from eight dyads of food-deprived nestlings recorded in 2008; two successive sequences of 40 calls were separated by a pause of $40 \mathrm{~s}$, and within each sequence, two successive calls were separated by a pause of $3 \mathrm{~s}$. This design gave enough time to the students to indicate whether two successive calls were produced by the same or different individual. In each of these eight sequences, each of the two nestlings emitted 20 calls each, selected randomly from 15 food-deprived recordings and inserted in a random order. Two successive calls in a sequence were emitted by the same individual 
in $50 \%$ of cases. Students, who never heard barn owls before the experiment, were informed that the aim was to determine whether human ear can discriminate without training calls from different barn owl nestlings. We explicitly told to the students that two individuals emitted the 40 calls of each series.

\section{Acoustic analyses}

Nestling dyads were recorded using two microphones (MC930; Beyerdynamic GmbH \& Co KG, Heilbronn, Germany) placed horizontally on the roof in the middle of the box and oriented in opposite directions, towards each nestling. We used Matlab (MathWorks, Natick, MA, USA) to assign calls to nestlings by comparing the signal level of the two microphones, because the microphone oriented towards a nestling that was calling gave a stronger signal than the microphone oriented in the opposite direction. We considered seven vocal parameters, the number of calls and six acoustic features measured using MATLAB (Dreiss et al., 2013a). The considered acoustic features were the following variables (see Fig. 1).

1 Call duration (s) of each single hissing call.

2 Absolute loudness level $(\mathrm{dB})$ of the calls, which is sensitive to the distance to the microphone, a variable that could not be recorded.

3 Loudness deviation [0-1]. This point divides the call in two parts in the time axis; the parts before and after this value represent half of call loudness $(\mathrm{dB})$. When loudness deviation is above 0.5, calls are louder at the end than at the beginning. It can be represented with the following formula, in which the signal segment containing one call is denoted $x(t)$, where $t$ is time in seconds. $T$ represents the call duration in seconds.

$$
\frac{\int_{0}^{T} t|x(t)|^{2} \mathrm{~d} t}{\int_{0}^{T}|x(t)|^{2} \mathrm{~d} t}
$$

4 Mean Frequency $(\mathrm{kHz})$. Frequencies above and below this value represent half of call loudness $(\mathrm{dB})$. It can be represented with the following formula, in which the spectrum of $x(t)$ is denoted $X(f)$, where $f$ is frequency in $\mathrm{Hz}$. The bandwidth of the signal is $F$ $\mathrm{Hz}$ :

$$
\frac{\int_{0}^{F} f|X(f)|^{2} \mathrm{~d} f}{\int_{0}^{F} f|X(f)|^{2} \mathrm{~d} f}
$$

5 Upper Frequency $(\mathrm{kHz})$. Frequencies above this value represent $25 \%$ of call loudness. It can be represented by the following formula:

$$
\frac{\int_{0}^{F} f^{2}|X(f)|^{2} \mathrm{~d} f}{\int_{0}^{F} f|X(f)|^{2} \mathrm{~d} f}
$$

6 Frequency variation $(\mathrm{kHz})$ represents the standard deviation of frequency with respect to time, which is computed after estimating mean frequency on shorttime spectra along the call.

In various types of systems, the pattern of frequency modulation of calls reveals identity (e.g. Janik et al., 2006), but as barn owl calls are quasi-stationary (i.e. frequencies are more or less constant from call beginning to the end), there is no such pattern.
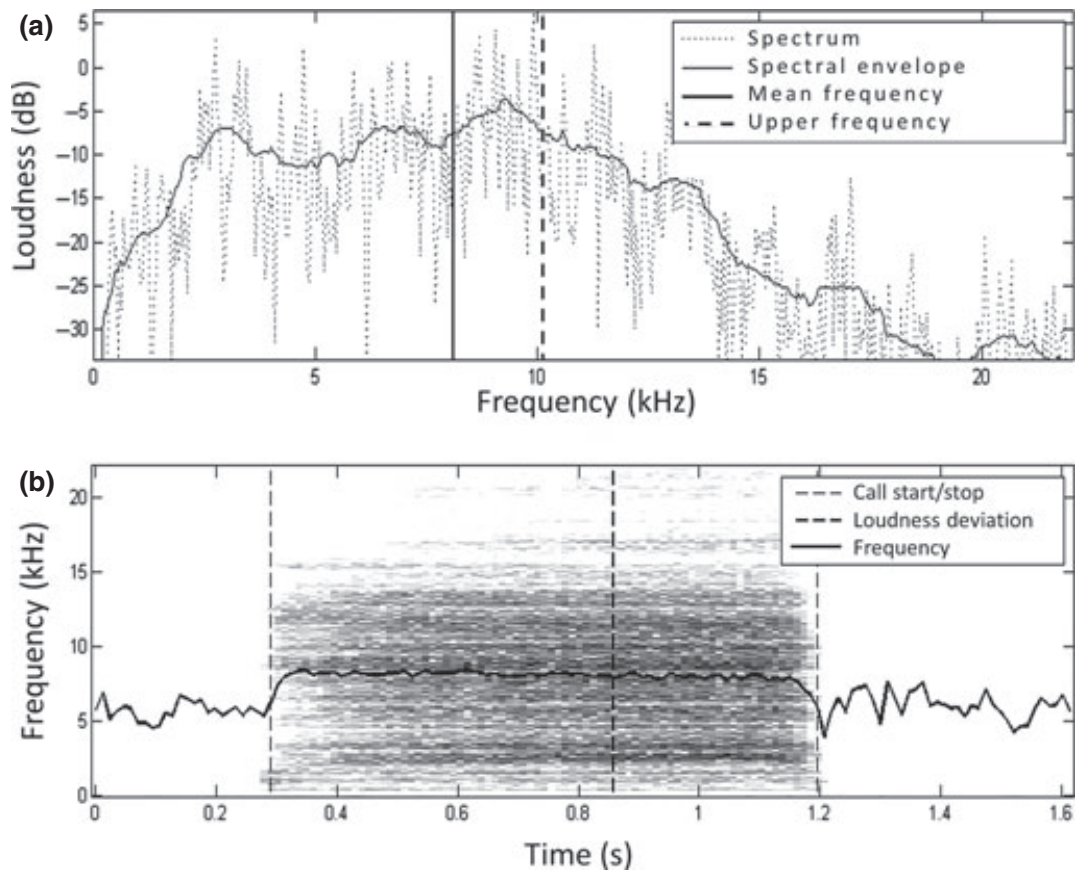

Fig. 1 Frequency distribution (a) and sonogram (b) of a typical negotiation call of a barn owl nestling. 


\section{Statistical analyses}

Statistical analyses were performed with the software SAS, v.9.1 (SAS Institute Inc., Cary, NC, USA). Distribution of acoustic parameters did not differ from normal distribution as confirmed by Kolmogorov-Smirnov tests. Number of calls was normalized with a logtransformation. Residuals of linear mixed models were normality distributed. Body condition of nestlings at capture was estimated with the scaled mass index (Body mass at capture $\left.\times(233 / \text { wing length at capure })^{0.57}\right)$, following Peig \& Green (2010), a size-independent condition indicator.

\section{Effects of absolute age, age hierarchy, sex and hunger on vocal parameters}

Linear mixed model with restricted maximum likelihood was used to determine the effect of food treatment, age hierarchy (i.e. junior vs. senior), absolute age and sex using for each individual an average value for each vocal parameter in each food treatment (food-satiated and food-deprived). As random factors, we set individual identity, dyad (nestmates recorded in the same box), nest of origin and nest of rearing, the latter two nested within the cross-foster group (i.e. two nests between which we swapped hatchlings belonged to the same cross-foster group). The statistical significances of random effects were estimated with likelihood ratio tests.

\section{Within-individual repeatability within each food treatment}

To examine the extent to which an individual produces calls that are consistently similar in their structure, we performed repeatability analyses according to Lessells and Boag (1987). We first examined whether the calls produced by an individual in a given food state (i.e. food-deprived or food-satiated) are significantly different from those produced by other individuals. For each of the 98 individuals, we randomly selected 10 calls of 30-3650 recorded calls for each food treatment, and hence, for each of the six vocal parameters, we tested whether these 10 calls are more similar to each other than to the calls produced by the 97 other individuals. We then tested whether within-individual repeatability of acoustic features differs when in a food-deprived compared with food-satiated state using Wilcoxon tests. This comparison was possible because repeatability was estimated with 10 different sets of calls and with the same sample size (10 calls) in each treatment.

Another approach to investigate whether calls are specific to each individual is to perform a parametric discriminant function analysis, to assess whether calls can be attributed to individuals based on the six acoustic features. This analysis groups calls by similarity and by comparing the classification of the discriminant analysis with the actual identity of the nestlings that produced these calls (i.e. classification error); we could estimate the extent to which we can statistically identify an individual based on call structure. Error rates were estimated by cross-validation, that is, each individual call was classified using a discriminant function computed from the other calls produced by the same individual (and calls produced by all other individuals), excluding the call being classified. To test the extent to which an individual can be statistically discriminated from other conspecifics based on its calls, we randomly selected 30 calls for each 98 individual and food treatment and estimated the individual discrimination error rate for each individual under a given food state.

\section{Within-individual repeatability across food treatments}

We performed another repeatability analysis to test whether an individual produces calls with a similar structure in the two food states. For each individual, we calculated two mean values per acoustic feature: one for all calls produced when food-deprived and one for all calls produced when food-satiated. We thus examined whether these two mean values are more similar to each other compared with mean values of the 97 other individuals.

To assess whether acoustic features provide individually distinct characteristics, we computed the 'potential for individual coding' (PIC) following Reers and Jacot (2011). To obtain a PIC value for a given acoustic feature, we estimated the individual coefficient of variations (CVi) for each individual using the average acoustic feature per food treatment and per individual, as well as the global $\mathrm{CV}$ of each acoustic feature (CVb) across all calls and all individuals, following the formula: $\mathrm{CV}=100 \times \mathrm{SD} /$ mean, with $\mathrm{SD}=$ standard deviation. The PIC value for each acoustic feature was obtained by computing the formula $\mathrm{PIC}=\mathrm{CVi} / \mathrm{CVb}$. Acoustic features with large compared with low PIC values are more distinct between individuals.

\section{Effects of nest of rearing and nest of origin on vocal parameters}

To estimate the heritability component of vocal parameters, we computed another sets of linear mixed models with restricted maximum likelihood using an average value per nestling, with sex and age hierarchy (i.e. junior vs. senior) as cofactors and absolute age as covariate. Heritability was estimated as the genetic variance divided by the phenotypic variance. The genetic variance was estimated as twice the variance of the nest of origin, as full siblings share half of their genes on average (Lynch \& Walsh, 1998). The phenotypic variance was estimated as the sum of the variance components of nest of origin, nest of rearing, dyad and the residual.

\section{Ethical note}

We brought to the university all but one nestling of each nest to ensure that parents do not abandon their 
nest. After 3 days and nights spent in the laboratory, nestlings were brought back to their nest of rearing. Nestlings were already thermo-independent and able to eat prey items by themselves. Nestlings in the laboratory were not physiologically stressed (Dreiss et al., 2010a) and fledged with similar success and with similar body mass than nonmanipulated nestlings (Dreiss et al., 2013a).

\section{Results}

\section{Covariation between vocal parameters}

Nestlings that emitted more calls produced longer and louder calls, which also tended to display lower mean and upper frequency (i.e. lower pitched) and to be louder at the end than beginning (i.e. larger loudness deviation; Table 1).

\section{Effects of absolute age, age hierarchy, sex and hunger on vocal parameters}

Among the six acoustic features, only call mean frequency $(\mathrm{kHz})$ was not significantly affected by food treatment (Table 2, Fig. 2a). When food-deprived, nestlings produced louder calls, less variable in frequency and more accentuated at the end than at the beginning of the calls compared with when food-satiated. Upper frequency was also lower in experimentally food-satiated nestlings, which means that the frequencies of calls above the mean frequency were lower pitched, making the call sounds lower. The strongest effect was observed for number of calls and call duration, as individuals produced $12 \%$ more calls and $11 \%$ longer in food-deprived than in food-satiated state, respectively (Fig. 2a; difference of vocal parameters between the two food treatments divided by average individual parameters calculated over the two food treatments;

\begin{tabular}{|c|c|c|c|c|c|c|}
\hline & $\begin{array}{l}\text { Call } \\
\text { duration }\end{array}$ & Loudness & $\begin{array}{l}\text { Loudness } \\
\text { deviation }\end{array}$ & $\begin{array}{l}\text { Mean } \\
\text { frequency }\end{array}$ & $\begin{array}{l}\text { Upper } \\
\text { frequency }\end{array}$ & $\begin{array}{l}\text { Frequency } \\
\text { variation }\end{array}$ \\
\hline $\begin{array}{l}\text { Number of } \\
\text { calls }\end{array}$ & $0.17^{\star}$ & $0.37^{\star \star \star}$ & $0.19^{*}$ & $-0.01^{\text {n.s. }}$ & $-0.15^{\text {n.s. }}$ & $-0.18^{\star}$ \\
\hline $\begin{array}{l}\text { Call } \\
\text { duration }\end{array}$ & & $0.42^{\star \star \star}$ & $0.37^{\star \star \star \star}$ & $0.04^{\text {n.s. }}$ & $-0.27^{\star *}$ & $-0.56^{\star \star \star}$ \\
\hline Loudness & & & $0.20^{\star}$ & $-0.23^{\star}$ & $-0.22^{\star}$ & $-0.19^{\star}$ \\
\hline $\begin{array}{l}\text { Loudness } \\
\text { deviation }\end{array}$ & & & & $0.09^{\text {n.s. }}$ & $-0.23^{\star}$ & $-0.11^{\text {n.s. }}$ \\
\hline $\begin{array}{l}\text { Mean } \\
\text { frequency }\end{array}$ & & & & & $0.70^{\star \star \star}$ & $0.36^{\star \star \star}$ \\
\hline $\begin{array}{l}\text { Upper } \\
\text { frequency }\end{array}$ & & & & & & $0.58^{\star \star \star}$ \\
\hline
\end{tabular}

Table 1 Pearson's correlation coefficient of number of calls and six acoustic parameters in barn owl nestlings, based on the average value per nestling $(N=146)$.

Values in bold considered significant after Bonferroni correction $(P<0.0002)$.

${ }^{*} P<0.05 ;{ }^{* *} P<0.002$ (level of significance after Bonferroni correction); $* * * P<0.0005$.

Table 2 Relationship between vocal parameters, food treatment, age hierarchy, absolute age and sex in barn owl nestlings. For each individual and vocal component, we calculated a mean value for each of the two recording sessions (food-deprived and food-satiated) which were used in linear mixed models (d.f. = 1,94). As random factors we set nestling identity, experimental dyad (nestlings were recorded in dyads of two nestmates), nests of origin and rearing nested in the cross-fostering group (cross-fostering was performed between pairs of nests). The statistical significances of random effects were estimated with likelihood ratio tests.

\begin{tabular}{|c|c|c|c|c|c|c|c|c|c|c|c|c|c|c|}
\hline \multirow[b]{2}{*}{ Effects } & \multicolumn{2}{|c|}{ Number of calls } & \multicolumn{2}{|c|}{$\begin{array}{l}\text { Call duration } \\
\text { (s) }\end{array}$} & \multicolumn{2}{|c|}{ Loudness (dB) } & \multicolumn{2}{|c|}{$\begin{array}{l}\text { Loudness } \\
\text { deviation }\end{array}$} & \multicolumn{2}{|c|}{$\begin{array}{l}\text { Mean } \\
\text { frequency }(\mathrm{kHz})\end{array}$} & \multicolumn{2}{|c|}{$\begin{array}{l}\text { Upper } \\
\text { frequency } \\
(\mathrm{kHz})\end{array}$} & \multicolumn{2}{|c|}{$\begin{array}{l}\text { Frequency } \\
\text { variation }(\mathrm{kHz})\end{array}$} \\
\hline & $F$ & $P$ & $F$ & $P$ & $F$ & $P$ & $F$ & $P$ & $F$ & $P$ & $F$ & $P$ & $F$ & $P$ \\
\hline Food treatment & 33.03 & $<.0001$ & 72.3 & $<.0001$ & 32.9 & $<.0001$ & 50.3 & $<.0001$ & 0.2 & 0.70 & 16.0 & 0.0001 & 6.4 & 0.013 \\
\hline Age hierarchy & 12.27 & 0.0007 & 9.2 & 0.0032 & 4.6 & 0.034 & 0.1 & 0.81 & 0.6 & 0.43 & 5.2 & 0.025 & 11.3 & 0.001 \\
\hline Absolute age & 3.35 & 0.07 & 0.1 & 0.81 & 0.3 & 0.60 & 0.1 & 0.84 & 12.15 & 0.0007 & 18.1 & $<.0001$ & 4.4 & 0.040 \\
\hline Sex & 3.36 & 0.07 & 0.2 & 0.70 & 0.5 & 0.48 & 0.6 & 0.44 & 21.19 & $<.0001$ & 6.5 & 0.012 & 1.6 & 0.21 \\
\hline Random: Ring & & 0.0005 & & $<.0001$ & & $<.0001$ & & $<.0001$ & & 0.009 & & $<.0001$ & & $<0.0001$ \\
\hline Random: Dyad & & 1 & & 0.09 & & 0.08 & & 1 & & 1 & & 1 & & 0.65 \\
\hline $\begin{array}{l}\text { Random: Nest of origin } \\
\text { (cross-fostering group) }\end{array}$ & & 0.13 & & 0.002 & & $<0.0001$ & & 0.009 & & 0.0009 & & 0.10 & & 0.24 \\
\hline $\begin{array}{l}\text { Random: Nest of rearing } \\
\text { (cross-fostering group) }\end{array}$ & & 1 & & 1 & & 0.40 & & 1 & & 1 & & 0.16 & & 0.07 \\
\hline
\end{tabular}



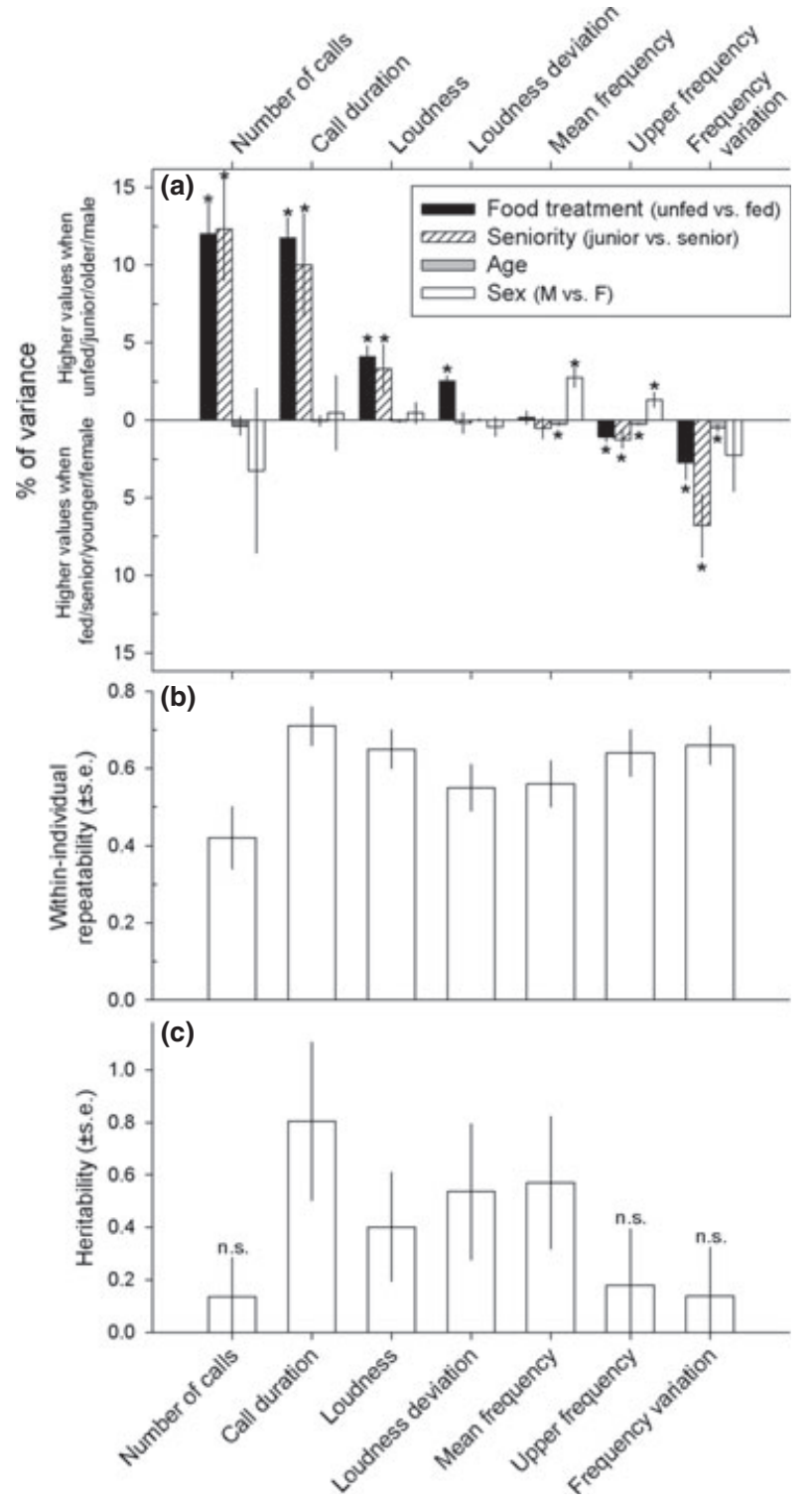

Fig. 2 (a) Percentage of variance of vocal parameters explained by food treatment (each barn owl nestling was recorded in an experimentally food-deprived and food-satiated state, the order of these manipulations between randomly allocated across nestlings), age hierarchy (nestlings were the senior (older) or the junior (younger) of the experimental dyads), absolute age and gender. Positive values indicate that nestlings display higher values of vocal parameters when unfed/junior/older/male than when fed/ senior/younger/female, and the opposite is true for negative values. (b) Repeatability (SE) of vocal parameters over the two nights of recordings and (c) heritability estimates (SE). ${ }^{*} \mathrm{p}<0.05$

loudness: 4\%, loudness deviation: 2\%, upper frequency: $1 \%$, frequency variation: $3 \%$ ).

Mean frequency and upper frequency decreased with absolute age (Table 2), older nestlings producing less variable and lower-pitched calls, when controlling for age hierarchy. Juniors produced more, longer, louder and lower-pitched calls than their senior siblings (Table 2, Fig. 2a), controlling for absolute age. Within calls, frequency variation was also lower in juniors than in seniors (Table 2, Fig. 2a).

Males produced calls about $0.2 \mathrm{kHz}$ higher in frequency than females (Table 2, Fig. 2a). The other vocal parameters did not differ significantly between the sexes. Female nestlings are slightly bigger than males (Roulin et al., 1999), but when controlling for sex, call frequency was not significantly related to body condition and wing length at capture (as a proxy of age and body size; same model as in Table 2 with body condition and wing length as additional covariates, mean frequency model: body condition: $F_{1,94}=1.25, P=0.27$; wing length: $F_{1,94}=1.55, P=0.22$; sex: $F_{1,94}=19.19$, $P<0.001$; upper frequency model: body condition: $F_{1,94}=2.78 ; P=0.10$; wing length: $F_{1,94}=3.66, P=$ 0.06; sex: $\left.F_{1,94}=6.90, P=0.01\right)$.

\section{Within-individual repeatability within each food treatment}

The 98 nestlings that vocalized both when fooddeprived and food-satiated produced between 30 and 3650 calls in each situation. Within-individual repeatability was significant for all vocal parameters, but did not differ between food-satiated and food-deprived treatments (all Wilcoxon tests $P$-values $>0.1$ in the comparison between the repeatability values estimated in food-satiated and food-deprived treatments, with 10 different sets of 10 calls per treatment; average \pm SE values for call duration: $0.63 \pm 0.03$; loudness: $0.59 \pm 0.03$; loudness deviation: $0.39 \pm 0.03$; mean frequency: $0.37 \pm 0.04$; upper frequency: $0.40 \pm 0.03$; frequency variation: $0.40 \pm 0.03)$. The statistical discrimination of the 98 individuals was higher when food-deprived (Wilcoxon signed rank test: $S=499.5$, $P=0.032$; discrimination success for food-deprived nestlings: $\quad 36.4 \pm 0.2 \%$; for food-satiated nestlings: $30.8 \pm 0.3 \%$; 61 individuals of 98 were more identifiable when food-deprived than food-satiated, which is more than at random in a binomial test: $P=0.015)$.

The relatively low success of discriminating individuals based on calls is explained by the fact that our statistics is based on 98 individuals which is biologically unrealistic (a nestling is surrounded by a maximum of eight nestmates). When we repeated the discriminant analyses among full siblings, the success of assigning calls correctly to a specific individual ranged from $90 \%$ for broods of two siblings (range is $75 \%$ and $98 \%$ ) to $62 \%$ for broods of six siblings (range is $56 \%$ and $70 \%$ ).

\section{Within-individual repeatability across food treatments}

Although most vocal parameters differed between food treatments, they were significantly repeatable across food 
treatments. Indeed, number of calls and the six acoustic features were significantly repeatable across food treatments (repeatability analysis on mean vocal parameters calculated for each individual in each food treatment and hence comparing two values per individual: number of calls, $r=0.42 \pm 0.08, F_{97,98}=2.17$; call duration, $r=$ $0.71 \pm 0.05, \quad F_{97,98}=4.64 ;$ loudness, $r=0.65 \pm 0.05$, $F_{97,98}=3.76$; loudness deviation, $r=0.55 \pm 0.06, F_{97,98}$ $=2.88$; mean frequency, $r=0.56 \pm 0.06, F_{97,98}=3.05$; upper frequency, $r=0.64 \pm 0.06, \quad F_{97,98}=4.20$; frequency variation, $r=0.66 \pm 0.05, \quad F_{97,98}=3.97$; all $P$-values $<0.0001, N=98$ individuals; Fig. $2 \mathrm{~b})$.

The PIC ratios of call duration and all acoustic features were $>1$. This indicates that we can successfully discriminate individuals using all seven vocal parameters, because the within-individual variability across food treatments is smaller than the interindividual variability (PIC for call duration: 2.14, loudness: 2.15, loudness deviation: 1.93, mean frequency: 1.91, upper frequency: 2.06, frequency variation: 2.17 ).

\section{Effects of nest of rearing and nest of origin on vocal parameters}

Four acoustic variables were significantly related to the nest of origin (Table 2, Figs 2c and 3; heritability estimates for call duration: $0.80 \pm 0.30$; loudness: 0.40 \pm 0.21 ; loudness deviation: $0.54 \pm 0.26$ and mean frequency: $0.57 \pm 0.25)$. The three other vocal parameters were not significantly related to the nest of origin (Table 2, Fig. 2c; heritability estimates for number of calls: $0.14 \pm 0.15$; upper frequency: $0.18 \pm 0.21$ and frequency variation: $0.14 \pm 0.19$ ). The heritable acoustic variables (call duration, loudness, mean frequency and loudness deviation) were not correlated with brood size in the nest of origin and body condition (similar mixed models as Table 2, with body mass at capture and brood size as covariates, $P$-values $>0.10)$. None of the vocal parameters were related to the nest of rearing (Table 2).

\section{Discrimination of barn owl calls by human}

When hearing successively eight dyads of nestlings of 15 possible ones (each nestling producing 20 calls), 19 human subjects could correctly assign calls to one of the two individuals in $71 \%$ of the cases, which is significantly higher than at random (binomial test: $P<0.00$ $01)$. Thus, each student correctly assigned on average 227 calls to one of the two individuals of the 312 calls he/she heard.

We then performed a discriminant analysis to test the extent to which the same 40 calls of the 15 different nestling dyads can also be statistically discriminated, using the six measured acoustic features. Human and statistical discrimination rates were positively correlated (Kendall correlation on average estimates of the 15 dyads: tau $b=0.57, P=0.005$, Fig. 4 ), showing that identifying individuals based on calls is easier when acoustic features are more differentiated.

\section{Discussion}

Our study shows that individual nestling barn owls consistently produce negotiation calls with a similar structure whatever the hunger state (within-individual repeatability values ranged from 0.42 to 0.71 ). Although these calls are sensitive to hunger level, they also reveal, in order of importance, identity, position in the within-brood age hierarchy, sex and absolute age.

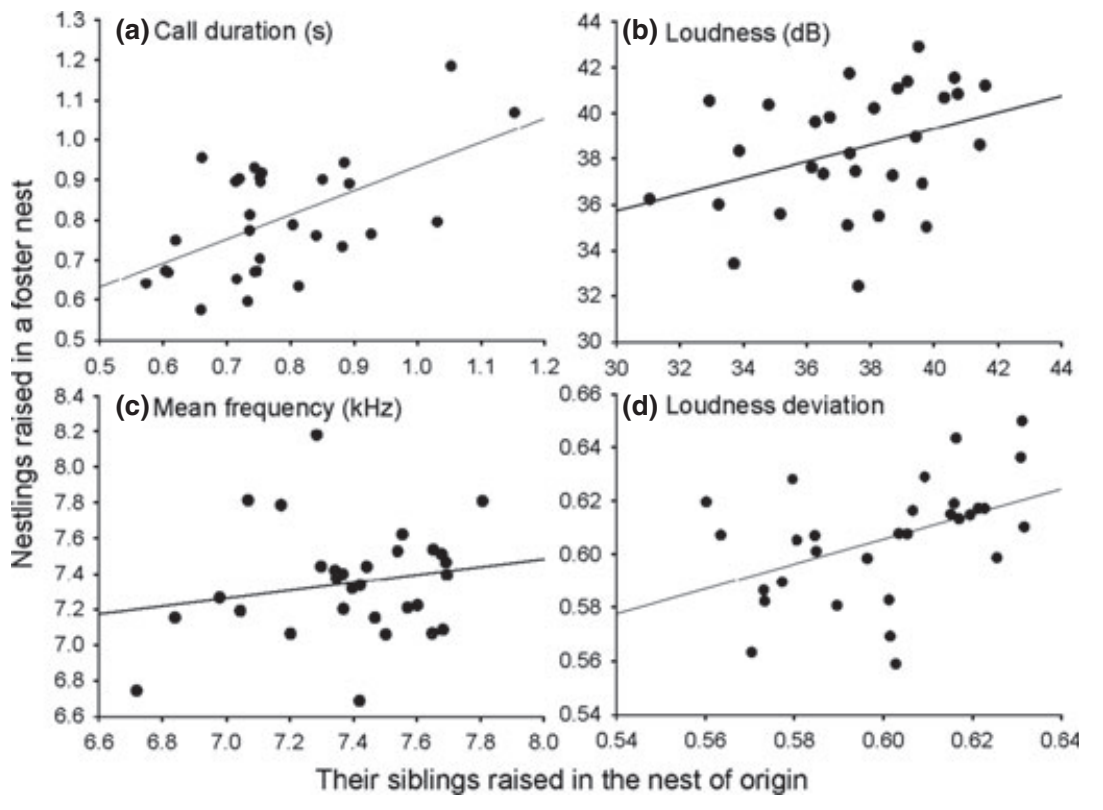

Fig. 3 Relationship between acoustic parameters of barn owl nestlings born in the same nest but raised in different nests for (a) call duration, (b) loudness, (c) mean frequency and (d) loudness deviation. Each dot represents the average value for a cross-fostering group. 


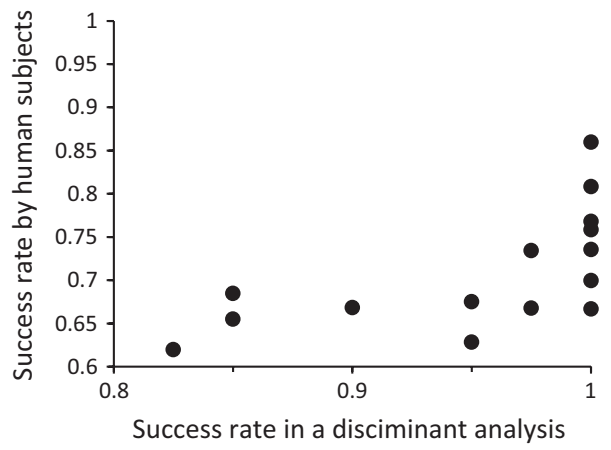

Fig. 4 Relationship between human and statistical successes in identifying which of two barn owl nestlings produced negotiation calls. Discriminant analysis was based on six acoustic features.

Interestingly, the nest of origin explains a large portion of the variance in vocal parameters (heritability from 0.4 to 0.8 ) although no call parameters are related to the nest of rearing.

\section{Efficiency of individual recognition}

Sibling recognition has been less studied than parentoffspring recognition (Nakagawa \& Waas, 2004; Scheiber et al., 2011) and is often presented as a way to differentiate kin from nonkin (Olsen et al., 1998). Therefore, to the best of our knowledge, researchers have not considered the possibility that sib-sib recognition mechanisms could permit the evolution of complex social interactions between siblings including the adjustment of behaviour according to short-term sibling interactions. Based on six acoustic features, a barn owl nestling could be statistically discriminated from its siblings with $62 \%$ and $90 \%$ of success for nests of 6 and 2 nestlings, respectively. Furthermore, human subjects listening to calls produced by one of two nestlings could discriminate which individual produced a given call in $71 \%$ of the cases. The positive correlation between the human ability to recognize nestling calls and statistical discrimination indicates that our chosen acoustic parameters are biologically relevant, some parameters being more reliable in identifying individuals than others (in order of importance call duration, loudness, frequency variation, upper frequency, loudness deviation and mean frequency). Taken together, our results suggest that barn owl nestlings might be able to recognize siblings individually. This ability should be facilitated by the fact that usually one barn owl nestling calls alone during long periods of time (Bühler \& Epple, 1980; Bunn et al., 1982) and nestlings actively avoid to call simultaneously as their nestmates (Dreiss et al., 2013b). This implies that nestlings usually hear every sibling call and thereby can perceive all acoustic features useful to recognize the identity of their siblings. Accordingly, we recently found that owlets adjust their behaviour according to the number of competitors that are currently vocalizing, implying that they determine whether successive calls are produced by the same or different individuals (Ruppli et al., 2013b). Furthermore, an individual differentially adjusts its behaviour depending on whether it is facing the dominant or the subordinate of a previously eavesdropped vocal interaction (i.e. which individual had called more rapidly after the other a minute ago; Dreiss et al., 2013a).

\section{Individual recognition: an adaptive role in sib-sib vocal negotiation?}

In the barn owl, the most vocal individual in the absence of parents induces its siblings to retreat from the contest once parents are back at the nest with a food item thereby facilitating the monopolization of this item (Roulin, 2002; Johnstone \& Roulin, 2003). The 'sibling negotiation hypothesis' relies on the assumption that negotiation calls produced in the absence of parents honestly signal their intention to compete for food at parents' return. Why should individuals signal their intention to compete to their siblings in the first place? Because food is not easily monopolized in front of a hungry rival, by signalling their motivation to compete, negotiating nestlings deter siblings from competing intensely at parent's return (Johnstone \& Roulin, 2003). Vocalizing at a lower rate would therefore induce an increase in the level of sibling competition at parent's return. If so, should individuals pretend to be hungrier than they actually are? Given that nestlings produce up to 5000 calls per night only in the absence of parents and that each call contains substantial noise energy (Bühler \& Epple, 1980), sibling negotiation is a process that is very likely to be costly. Furthermore, because barn owl nestmates are full siblings, each nestling has indirect fitness gains if their siblings obtain enough food and hence if food is fairly distributed according to need (Roulin et al., 2012).

Because negotiation in parent's absence last hours and nestlings produce in total more calls in this phase than during parent-offspring communication, it should more honestly reveals need than begging towards parents, as demonstrated in a previous study (Roulin, 2001). To verify that food obtaining is the outcome of negotiation process in parents' absence and hence is fairly distributed according to need, nestlings should be able to assign each single call to the sibling that emitted it. This aspect of signal honestly has been rarely considered, and for this reason, we elaborate this speculative issue. We propose that individual recognition is necessary for a sib-sib communication system to be evolutionary stable. Two arguments plead for this interpretation. First, an individual would likely benefit from being vocally recognizable, so that its siblings perceive all the calls it emitted and assign them to itself and not to other siblings. In a dark crowded nest, vocal 
signature could be particularly important as individual position in the nest is likely to give little information on the caller identity. Second, thanks to an individual recognition mechanism, nestmates can verify that the individual that obtains the food at parent return is the same as the one that previously negotiated at the highest level with its siblings. This ensures that the food is fairly distributed according to the previously witnessed individual level of calling during the sibling negotiation phase. A 'cheater' that monopolizes a food item without having negotiated beforehand may be 'punished' in different ways. For instance, its siblings (i.e. the one that previously negotiated at the highest level but also its siblings) may physically punish it (Clutton-brock $\delta$ Parker, 1995), something that is unlikely to occur in the barn owl, a species in which aggressive sib-sib interactions are very rarely observed (Bunn et al., 1982; Roulin et al., 2012). Another form of punishment is to steal the food item that a cheater just obtained, a behaviour that is frequently observed in the barn owl (Bühler, 1981, Bunn et al., 1982; Roulin et al., 2008; Roulin et al., 2012).

Nestling individual discrimination was significantly higher in a food-deprived than food-satiated state $(36 \%$ of success vs. $31 \%$ ). This is consistent with a previous study in Jackson's golden-backed weavers (Ploceus jacksoni) showing that chicks were more easily identifiable statistically when begging in a hungry than in satiated state (Reers \& Jacot, 2011). Using the same argumentation as above, this finding is not surprising. Under the hypothesis that nestlings use calls to recognize siblings and assess their vocal performance before deciding whether they should enter into the competition or not, it seems particularly beneficial for individual nestlings to be recognizable when hungry. Because the next prey item is highly valuable for hungry nestlings and they invest more effort in calling than food-satiated nestlings, they would derive more benefits from being identified as motivated competitors to be given the priority to the prey. Alternatively, the change in discriminability with hunger level could be a nonadaptive by-product, due to the fact that nestlings' motivation is more variable when food-satiated, and they signal at maximal and stable level when food-deprived (Reers $\delta$ Jacot, 2011). This argument does not apply to the barn owl, as we showed that the acoustic features signalling hunger level (such as call duration) present similar within-individual repeatability when food-deprived and food-satiated.

\section{Recognizing individuals over repeated interactions: adjustment to individual characteristics?}

In the above discussion, we suggested that individual recognition is important during a single competitive event, for instance in the barn owl from the moment when siblings start to negotiate up to when parents actually deliver food. Here, we speculate that selection should also favour individuals to remember the identity of conspecifics or siblings during longer periods of time and, furthermore, to associate identity with individual characteristics that are stable over time such as personality (Sih et al., 2004) and resource-holding potential. Indeed, age differences between barn owl siblings are pronounced, implying that the level of individual motivation is only partly reflected by the number and the quality of emitted calls. Accordingly, we have shown here that seniors produce fewer negotiation calls than juniors independently of hunger level (see also Dreiss et al., 2010b). Hence, to accurately estimate the level of a nestmate's motivation to compete, nestlings should calibrate their signalling level with the signaller's characteristics, such as position in the within-brood age hierarchy, sex or personality. Furthermore, competing with an older or intrinsically bolder individual is surely less rewarding than competing with a younger or peaceful sibling, even if they present similar food need (Roulin et al., 2010). Our study system is consistent with this scenario because nestlings modulate the number of emitted negotiation calls according to whether they face the senior or the junior of an eavesdropped interaction between two siblings (Dreiss et al., 2013a). The latter study strongly suggests that nestlings can identify which of two nestmates is the oldest based on acoustic features, which is in line with the present study showing that vocal parameters were related to age hierarchy independently of absolute age and body condition. This raises the question of whether the development of call features associated with age hierarchy is influenced by social interactions, something that remains to be tackled.

\section{Calling features signalling food need, age and sex}

Food treatment influenced to large extent vocal parameters related to vocal performance, such as call duration, number of calls or call loudness than parameters related to sound pitch. The stronger influence of hunger level appeared to be on number of calls and call duration, two parameters for which variation is closely associated with the probability of food obtaining at parental return (Dreiss et al., 2010b). However, hunger also significantly affected call structure, such as withincall loudness deviation, call upper frequency and frequency variation. Similarly, in the presence of an older sibling, nestlings produced a higher performance signal (more, longer and louder calls) and lower-pitched calls, maybe as a mean to compensate for a low ranking position. In barn owls, call frequency slightly decreases with age, and nestlings seem to be able to perceive this difference in frequency. Indeed, we showed that nestlings finely adjust their vocal behaviour according to the age hierarchy of vocal competitors showing a difference of only a few days and retreat in front of older 
competitors (Dreiss et al., 2013a). Therefore, lowering call frequency when hungry and when facing an older sibling might be a mean to increase the likelihood of outcompeting siblings. This is in line with studies performed in other bird species showing that call frequency is lowered when motivation increases (e.g. Morton, 1977; Geberzahn et al., 2009), to reach signal frequency typically produced by bigger and highly competitive individuals (Davies \& Halliday, 1978). The pattern is reversed in some species in which call frequency increases with need (e.g. Marques et al., 2009), as higher frequency could be a physiological consequence of calling louder (Nemeth \& Brumm, 2010).

We would expect that signalling parameters that are more sensitive to variation in food need to play a less important role in individual signature than less sensitive factors (Dale et al., 2001). In our study, this was true for the number of emitted calls, which greatly varied with food treatment and was less repeatable across treatments and within individual than other vocal parameters. However, call duration - and to a lower extent loudness, upper frequency, frequency variation and loudness deviation - showed high within-individual repeatability and were nevertheless affected by food treatment. Moreover, although mean frequency was the only parameter not significantly related to food treatment, this parameter was less repeatable within individual than call duration or loudness. Therefore, contrary to expectation (Dale et al., 2001), information on individuality can be coded with multiple traits that can be sensitive to body condition and the environment, as also shown in the begging calls of Jackson's golden-backed weavers (Reers \& Jacot, 2011). Further studies are needed to determine whether the vocal signature is stable in time, as acoustic features slightly varied with age, and we recorded individuals during only two successive days. Calls can indeed be modulated during development (Leonard \& Horn, 2006; Marques et al., 2010).

The slight sex difference we found on some barn owl vocal parameters is unlikely to be due to size, for which we controlled for in the statistical analyses, but may be related to hormonal differences or sex-linked genes (Tomaszycki et al., 2001; Saino et al., 2003; Cynx et al., 2005). It would be interesting to further test whether nestlings recognize the sex of siblings and whether it influences sibling competition. In a different social system, the hyena Crocuta crocuta (Golla et al., 1999), competition between same-sex siblings is more intense than between male and female siblings.

\section{Effect of nest of origin on calling features}

Several studies showed a significant heritability of nestling bird begging displays (Kölliker et al., 2000; Dor \& Lotem, 2009; Kim et al., 2011). In nestling barn owls, four vocal parameters (call duration, loudness, loudness deviation and mean frequency) were related to the nest of origin. Because call duration, loudness and loudness deviation were slightly correlated, they may in part be related to the same genetic, maternal (Eising $\&$ Groothuis, 2003) or early development factors (MacDonald et al., 2006). Of note, the effect of nest of origin did not prevent full siblings to be vocally distinct, indicating that even if siblings call similarly, there is nevertheless ample variation in call structure between siblings to discriminate them. The effect of the nest of origin on vocalization was not due to the fact that full siblings share a common brood size in the nest of birth or because they have a similar size, which could reveal the quality of early development. To our surprise, no vocal parameters were significantly related to the nest of rearing and the dyad in which nestlings were recorded, although barn owl nestlings finely adjust their number of calls and call duration to their nestmates' vocal production (Roulin et al., 2009; Ruppli et al., 2013a). The nest of rearing can also be related to parent feeding methods or environmental conditions such as temperature, habitat or nest microhabitat, which could influence vocal learning or vocal production through neurological, physiological or morphological traits (Cynx et al., 2005; Dor \& Lotem, 2009). Hence, although vocal parameters fluctuates in time according to surrounding social conditions in this species, average values of vocal parameters appear to be only dependent of the genetic origin. It now remains to investigate whether these origin-related effects on vocalizations have an adaptive function and also to examine the evolutionary consequences (Kölliker et al., 2000).

\section{Acknowledgments}

We thank C. Faller and I. Henry for their help and two anonymous reviewers for comments on this manuscript.

\section{References}

Aubin, T. \& Jouventin, P. 1998. Cocktail-party effect in king penguin colonies. Proc. R. Soc. B-Biol. Sci. 265: 1665-1673.

Boncoraglio, G., Caprioli, M. \& Saino, N. 2009. Fine-tuned modulation of competitive behaviour according to kinship in barn swallow nestlings. Proc. R. Soc. B-Biol. Sci. 276: $2117-$ 2123.

Bühler, P. 1981. Das Fütterungsverhalten der Schleiereule (Tyto alba). Ökologie der Vogel 3: 181-183.

Bühler, P. \& Epple, W. 1980. Die Lautäußerungen der Schleiereule (Tyto alba). J. Ornithol. 121: 36-70.

Bunn, D.S., Warburton, A.B. \& Wilson, R.D.S. 1982. The Barn Owl. Poyser, London.

Christe, P., Richner, H. \& Oppliger, A. 1996. Begging, food provisioning, and nestling competition in great tit broods infested with ectoparasites. Behav. Ecol. 7: 127-131. 
Clutton-Brock, T.H. \& Parker, G.A. 1995. Punishment in animal societies. Nature 373: 209-216.

Cynx, J., Bean, N.J. \& Rossman, I. 2005. Testosterone implants alter the frequency range of zebra finch songs. Horm. Behav. 47: 446-451.

Dale, J., Lank, D.B. \& Reeve, H.K. 2001. Signaling individual identity versus quality: a model and case studies with ruffs, queleas, and house finches. Am. Nat. 158: 75-86.

Davies, N.B. \& Halliday, T.R. 1978. Deep croaks and fighting assessment in toads Bufo bufo. Nature 274: 683-685.

Dor, R. \& Lotem, A. 2009. Heritability of nestling begging intensity in the house sparrow (Passer domesticus). Evolution 63: $738-748$.

Draganoiu, T.I., Nagle, L., Musseau, R. \& Kreutzer, M. 2006. In a songbird, the black redstart, parents use acoustic cues to discriminate between their different fledglings. Anim. Behav. 71: 1039-1046.

Dreiss, A.N., Henry, I., Ruppli, C., Almasi, B. \& Roulin, A. 2010a. Darker eumelanic barn owls better withstand food depletion through resistance to food deprivation and lower appetite. Oecologia 164: 65-71.

Dreiss, A.N., Lahlah, N. \& Roulin, A. 2010b. How siblings adjust sib-sib communication and begging signals to each other. Anim. Behav. 80: 1049-1055.

Dreiss, A.N., Ruppli, C.A., Faller, C. \& Roulin, A. 2013a. Big brother is watching you: eavesdropping to resolve family conflicts. Behav. Ecol. 24: 717-722.

Dreiss, A.N., Ruppli, C.A., Oberli, F., Antoniazza, S., Henry, I. \& Roulin, A. 2013b. Barn owls do not interrupt their siblings. Anim. Behav. 86: 119-126.

Eising, C.M. \& Groothuis, T.G.G. 2003. Yolk androgens and begging behaviour in black-headed gull chicks: an experimental field study. Anim. Behav. 66: 1027-1034.

Forstmeier, W., Burger, C., Temnow, K. \& Deregnaucourt, S. 2009. The genetic basis of Zebra finch vocalizations. Evolution 63: 2114-2130.

Geberzahn, N., Goymann, W., Muck, C. \& ten Cate, C. 2009. Females alter their song when challenged in a sex-role reversed bird species. Behav. Ecol. Sociobiol. 64: 193-204.

Golla, W., Hofer, H. \& East, M.L. 1999. Within-litter sibling aggression in spotted hyaenas: effect of maternal nursing, sex and age. Anim. Behav. 58: 715-726.

Holley, A.J.F. 1984. Adoption, parent-chick recognition and maladaption in the Herring Gull Larus argentatus. Zeitschrift für Tierpsychologie 64: 9-14.

Janik, V.M., Sayigh, L.S. \& Wells, R.S. 2006. Signature whistle shape conveys identity information to bottlenose dolphins. Proc. Natl. Acad. Sci. USA 103: 8293-8297.

Janik, V.M. \& Slater, P.J.B. 2000. The different roles of social learning in vocal communication. Anim. Behav. 60: 1-11.

Jenkins, P.F. 1978. Cultural transmission of song patterns and dialect development in a free-living bird population. Anim. Behav. 26: 50-78.

Johnstone, R.A. \& Roulin, A. 2003. Sibling negotiation. Behav. Ecol. 14: 780-786.

Kedar, H., Rodriguez-Girones, M.A., Yedvab, S., Winkler, D.W. \& Lotem, A. 2000. Experimental evidence for offspring learning in parent- offspring communication. Proc. R. Soc. B-Biol. Sci. 267: 1723-1727.

Kempenaers, B. \& Sheldon, B.C. 1996. Why do male birds not discriminate between their own and extra-pair offspring? Anim. Behav. 51: 1165-1173.
Kim, S.Y., Noguera, J.C., Morales, J. \& Velando, A. 2011. The evolution of multicomponent begging display in gull chicks: sibling competition and genetic variability. Anim. Behav. 82: 113-118.

Kölliker, M., Brinkhof, M.W.G., Heeb, P., Fitze, P.S. \& Richner, H. 2000. The quantitative genetic basis of offspring solicitation and parental response in a passerine bird with biparental care. Proc. R. Soc. B-Biol. Sci. 267: 2127-2132.

Leonard, M.L. \& Horn, A.G. 2006. Age-related changes in signalling of need by nestling tree swallows (Tachycineta bicolor). Ethology 112: 1020-1026.

Leonard, M.L., Horn, A.G. \& Parks, E. 2003. The role of posturing and calling in the begging display of nestling birds. Behav. Ecol. Sociobiol. 54: 188-193.

Lessells, C.M. \& Boag, P.T. 1987. Unrepeatable repeatabilities: a common mistake. Auk 104: 116-121.

Levrero, F., Durand, L., Vignal, C., Blanc, A. \& Mathevon, N. 2009. Begging calls support offspring individual identity and recognition by zebra finch parents. C.R. Biol. 332: 579-589.

Lind, H., Dabelsteen, T. \& McGregor, P.K. 1996. Female great tits can identify mates by song. Anim. Behav. 52: 667-671.

Lynch, M. \& Walsh, B. 1998. Genetics and Analysis of Quantitative Traits. Sinauer, Sunderland, MA.

MacDonald, I.F., Kempster, B., Zanette, L. \& MacDougall-Shackleton, S.A. 2006. Early nutritional stress impairs development of a song-control brain region in both male and female juvenile song sparrows (Melospiza melodia) at the onset of song learning. Proc. R. Soc. B-Biol. Sci. 273: 2559-2564.

Marques, P.A.M., Vicente, L. \& Marquez, R. 2009. Nestling begging call structure and bout variation honestly signal need but not condition in Spanish sparrows. Zool. Stud. 48: 587-595.

Marques, P.A.M., de Araujo, C.B. \& Vicente, L. 2010. Nestling call modification during early development in a colonial passerine. Bioacoustics 20: 45-58.

Medvin, M.B., Stoddard, P.K. \& Beecher, M.D. 1992. Signals for parent-offspring recognition - strong sib-sib call similarity in cliff swallows but not barn swallows. Ethology 90: 17-28.

Morton, E.S. 1977. On the occurrence and significance of motivation-structural rules in some bird and mammal sounds. Am. Nat. 111: 855-869.

Naguib, M. \& Todt, D. 1997. Effects of dyadic vocal interactions on other conspecific receivers in nightingales. Anim. Behav. 54: 1535-1543.

Nakagawa, S. \& Waas, J.R. 2004. 'O sibling, where art thou?' - a review of avian sibling recognition with respect to the mammalian literature. Biol. Rev. 79: 101-119.

Nemeth, E. \& Brumm, H. 2010. Birds and anthropogenic noise: are urban songs adapti. Am. Nat. 176: 465-475.

Olsen, K.H., Grahn, M., Lohm, J. \& Langefors, A. 1998. MHC and kin discrimination in juvenile Arctic charr, Salvelinus alpinus (L.). Anim. Behav. 56: 319-327.

Payne, R.J.H. \& Pagel, M. 1997. Why do animals repeat displays? Anim. Behav. 54: 109-119.

Peig, J. \& Green, A.J. 2010. The paradigm of body condition: a critical reappraisal of current methods based on mass and length. Funct. Ecol. 24: 1323-1332.

Quine, D.B. \& Konishi, M. 1974. Absolute frequency discrimination in barn owl. J. Comp. Physiol. 93: 347-360.

Reers, H. \& Jacot, A. 2011. The effect of hunger on the acoustic individuality in begging calls of a colonially breeding weaver bird. BMC Ecol. 11: 3. 
Roulin, A. 2001. Food supply differentially affects sibling negotiation and competition in the barn owl (Tyto alba). Behav. Ecol. Sociobiol. 49: 514-519.

Roulin, A. 2002. The sibling negotiation hypothesis. In: The Evolution of Begging: Competition, Cooperation and Communication (J. Wright, M.L. Leonard, eds), pp. 107-127. Kluwer Academic Press, Dordrecht.

Roulin, A. 2004. The function of food stores in bird nests: observations and experiments in the barn owl Tyto alba. Ardea 92: 69-78.

Roulin, A., Colliard, C., Russier, F., Fleury, M. \& Grandjean, V. 2008. Sib-sib communication and the risk of prey theft in the barn owl Tyto alba. J. Avian Biol. 39: 593-598.

Roulin, A., Da Silva, A. \& Ruppli, C.A. 2012. Dominant nestlings displaying female-like melanin coloration behave altruistically in the barn owl. Anim. Behav. 84: 12291236.

Roulin, A., Dreiss, A.N., Fioravanti, C. \& Bize, P. 2009. Vocal sib-sib interactions: how siblings adjust signalling level to each other. Anim. Behav. 77: 717-725.

Roulin, A., Dreiss, A.N. \& Kolliker, M. 2010. Evolutionary perspective on the interplay between family life, and parent and offspring personality. Ethology 116: 787-796.

Roulin, A., Ducrest, A.L. \& Dijkstra, C. 1999. Effect of brood size manipulations on parents and offspring in the barn owl Tyto alba. Ardea 87: 91-100.

Roulin, A., Muller, W., Sasvari, L., Dijkstra, C., Ducrest, A.L., Riols, C. et al. 2004. Extra-pair paternity, testes size and testosterone level in relation to colour polymorphism in the barn owl Tyto alba. J. Avian Biol. 35: 492-500.
Ruppli, C.A., Dreiss, A.N. \& Roulin, A. 2013a. Efficiency and significance of multiple vocal signals in sibling competition. Evol. Biol. doi: 10.1007/s1 1692-013-9233-8.

Ruppli, C.A., Dreiss, A.N. \& Roulin, A. 2013b. Nestling barn owls assess short-term variation in the amount of vocally competing siblings. Anim. Cogn. 16: 993-1000.

Saino, N., Galeotti, P., Sacchi, R., Boncoraglio, G., Martinelli, R. \& Moller, A.P. 2003. Sex differences in begging vocalizations of nestling barn swallows, Hirundo rustica. Anim. Behav. 66: 1003-1010.

Scheiber, I.B.R., Hohnstein, A., Kotrschal, K. \& Wei $\beta$, B.M. 2011. Juvenile Greylag geese (Anser anser) discriminate between individual siblings. PLOS ONE 6: e22853.

Sih, A., Bell, A. \& Johnson, J.C. 2004. Behavioral syndromes: an ecological and evolutionary overview. Trends Ecol. Evol. 19: 372-377.

Stoddard, P.K., Beecher, M.D., Horning, C.L. \& Campbell, S.E. 1991. Recognition of individual neighbors by song in the song sparrow, a species with song repertoires. Behav. Ecol. Sociobiol. 29: 211-215.

Tibbetts, E.A. \& Dale, J. 2007. Individual recognition: it is good to be different. Trends Ecol. Evol. 22: 529-537.

Tomaszycki, M.L., Davis, J.E., Gouzoules, H. \& Wallen, K. 2001. Sex differences in infant rhesus macaque separation-rejection vocalizations and effects of prenatal androgens. Horm. Behav. 39: 267-276.

Received 19 July 2013; revised 17 September 2013; accepted 3 October 2013 